\title{
Signatures of sterile neutrino oscillations in high-energy cosmic neutrino flux
}

\section{Osamu Yasuda*}

Department of Physics, Tokyo Metropolitan University, Hachioji, Tokyo 192-0397, Japan

E-mail: yasuda AT phys.metro-u.ac.jp

\section{Andrea Donini}

Instituto de Física Teórica UAM/CSIC, Cantoblanco, E-28049 Madrid, Spain

E-mail: andrea.donini AT uam.es

\begin{abstract}
We discuss the possible effects of a (3+1)-scheme within the MiniBooNE constraint on the flavor ratio of high-energy cosmic neutrinos from cosmologically distant astrophysical sources. It is shown that the $v_{\mu}-v_{\tau}$ ratio is relatively insensitive to the theoretical uncertainties and therefore this ratio is the key ingredient to look for the signatures of the sterile neutrino scheme.
\end{abstract}

10th International Workshop on Neutrino Factories, Super beams and Beta beams

June 30 - July 52008

Valencia, Spain

* Speaker. 
High energy cosmic neutrinos from cosmologically distant astrophysical sources, such as Active Galactic Nuclei (AGN) or Gamma Ray Bursts (GRB) have attracted much attention because ongoing or future experiments are expected to observe them. Observation of these high energy neutrinos has advantage because low backgrounds of atmospheric neutrinos are expected and it is believed that e, $\mu, \tau$ flavors can be distinguished for energy higher than $10^{6} \mathrm{GeV}$ by looking for shower events, muon track events and "double bang events" [1], respectively.

It has been known [1] that, if the initial flavor ratio of the neutrino flux is $\Phi_{e}^{0}: \Phi_{\mu}^{0}: \Phi_{\tau}^{0}=$ $(1: 2: 0)$ which is expected from pion decays, then the ratio observed on the Earth becomes $\Phi_{e}$ : $\Phi_{\mu}: \Phi_{\tau}=(1: 1: 1)$ in the standard three flavor framework with oscillation parameters $\theta_{23} \simeq \pi / 4$, $\left|\theta_{13}\right| \ll 1$. In Ref. [2] possible deviation of the flavor ratio from that of the three flavor case was studied for a sterile neutrino scenario called the $(2+2)$-scheme, which was allowed at that time. The sterile neutrino scenarios, which were mainly introduced to account for the LSND anomaly [3] in terms of neutrino oscillations, is now strongly disfavored (see, e.g., Ref. [4]) irrespective of whether the mass pattern is of $(2+2)$ or $(3+1)$ type.

However, if we do not impose the LSND data, then we can still have sterile neutrino scenarios which are consistent with all the negative experimental data. Since it is known that $(2+2)$-schemes are inconsistent with combined results of the solar and atmospheric neutrino data, the only possibility is (3+1)-schemes whose mixings are small enough to satisfy all the experimental results except LSND. In fact, such a scenario was studied in Ref. [5] and the present bound on the mixing angles of a (3+1)-scheme was given.

Taking into consideration of the allowed regions for the mixing angles which were obtained in Ref. [5], deviation of the flavor ratio from $\Phi_{e}: \Phi_{\mu}: \Phi_{\tau}=(1: 1: 1)$ was studied [6]. The result is depicted in Fig.1 (a).

On the other hand, it was pointed out in Ref. [7] that there are theoretical uncertainties in the predictions for the flavor ratio and also an energy dependence is induced in the neutrino flavor fluxes. To illustrate their point, a detailed discussion of the energy dependence of neutrino flavor fluxes originated from GRB was given in Ref. [7] based on the model by Waxman and Bahcall [8]. Using the results in Ref. [7], it was found in Ref. [6] that the flavor ratio $\Phi_{e} / \Phi_{\mu}$ suffers strongly from energy dependence while the energy dependence of the ratio $\Phi_{\tau} / \Phi_{\mu}$ is small (see Fig 1 (b)(e)), so the ratio $\Phi_{\tau} / \Phi_{\mu}$ is the key ingredient to look for the signatures of the sterile neutrino scheme.

Statistical requirements to look for the signatures of the sterile neutrino oscillations were also discussed in Ref. [6]. After 10 years of data taking at a $\mathrm{km}^{3}$ detector, it is expected that we get $O(100) v_{e}$ and $v_{\mu}$ events and $O(30) v_{\tau}$ events from GRB's. Introducing the notations for the flavor ratios of the number of events $r_{e \mu} \equiv\left(N_{e}+\bar{N}_{e}\right) /\left(N_{\mu}+\bar{N}_{\mu}\right)$ and $r_{\tau \mu} \equiv\left(N_{\tau}+\bar{N}_{\tau}\right) /\left(N_{\mu}+\bar{N}_{\mu}\right)$, we found that the expected flavor ratios $r_{\tau \mu}$ for three and four family schemes are $r_{\tau \mu}^{(3-\text { fam })} \simeq 0.30 \pm$ 0.03 (theo) \pm 0.06 (stat) and $r_{\tau \mu}^{(4-\mathrm{fam})} \geq \min \left(r_{\tau \mu}^{(4-\mathrm{fam})}\right) \simeq 0.2$, respectively. Thus, as far as neutrinos from GRB's are concerned, statistics at a $\mathrm{km}^{3}$ detector are unfortunately not large enough to look for the signatures of the sterile neutrino. However, if other sources such as AGN's are found with a bigger neutrino flux, there may be a chance for the next generation of neutrino telescopes to observe or to constrain the four-family mixing model. 

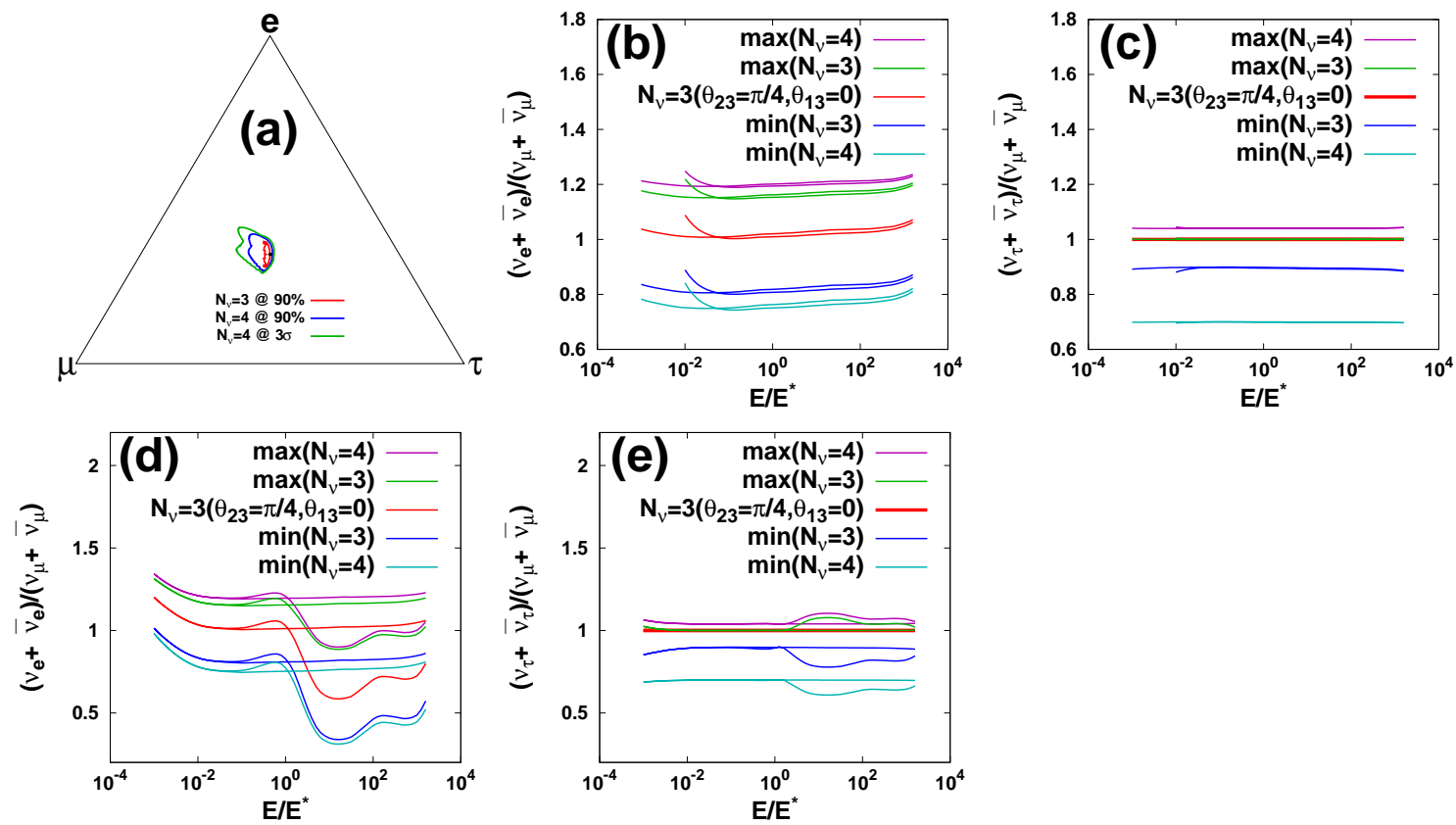

Figure 1: (a): The allowed region of the flavor ratio $\Phi_{e}: \Phi_{\mu}: \Phi_{\tau}$ in the triangle representation [2] for the three flavor case (bounded by the red thin line) and the (3+1)-scheme at 90\% CL (bounded by the blue thick line; the allowed region at $3 \sigma \mathrm{CL}$ is bounded by the green line). The standard ratio of the initial flux $\Phi_{e}^{0}: \Phi_{\mu}^{0}: \Phi_{\tau}^{0}=1: 2: 0$ is assumed. (b),(c): The observed ratios $\left(v_{e}+\bar{v}_{e}\right) /\left(v_{\mu}+\bar{v}_{\mu}\right)$ and $\left(v_{\tau}+\bar{v}_{\tau}\right) /\left(v_{\mu}+\bar{v}_{\mu}\right)$ for neutrinos from a GRB source as functions of the neutrino energy when the two sets of the reference values $\left(\alpha=2, \beta_{1}=1, \beta_{2}=2.4\right)$ and $\left(\alpha=2.4, \beta_{1}=1, \beta_{2}=2\right)$ are taken for the parameters $\alpha$ and $\beta$ in the proton and photon energy spectrum (cf. Fig. 14 of Ref. [7]). The five cases (the three flavor case with the best-fit oscillation parameters; the three flavor cases which give the maximum and minimum ratios; the four flavor cases which give the maximum and minimum ratios) are considered for each reference parameter set. (d),(e): The same as (b),(c) when the effect of the synchrotron losses of high energy muons are taken into account with the reference value $\varepsilon_{\mu}=\infty$ and 3 , where $\varepsilon_{\mu}$ is a parameter which characterizes the magnetic field (cf. Fig. 15 of Ref. [7]).

\section{References}

[1] J. G. Learned and S. Pakvasa, Astropart. Phys. 3, 267 (1995) [arXiv:hep-ph/9405296].

[2] H. Athar, M. Jezabek and O. Yasuda, Phys. Rev. D 62, 103007 (2000) [arXiv:hep-ph/0005104].

[3] C. Athanassopoulos et al. [LSND Collaboration], Phys. Rev. Lett. 77, 3082 (1996) [arXiv:nucl-ex/9605003].

[4] M. Maltoni, T. Schwetz, M. A. Tortola and J. W. F. Valle, New J. Phys. 6, 122 (2004). updated results in hep-ph/0405172 (v6).

[5] A. Donini, M. Maltoni, D. Meloni, P. Migliozzi and F. Terranova, JHEP 0712, 013 (2007) [arXiv:0704.0388 [hep-ph]].

[6] A. Donini and O. Yasuda, arXiv:0806.3029 [hep-ph].

[7] P. Lipari, M. Lusignoli and D. Meloni, Phys. Rev. D 75, 123005 (2007) [arXiv:0704.0718 [astro-ph]].

[8] E. Waxman and J. N. Bahcall, Phys. Rev. Lett. 78, 2292 (1997) [arXiv:astro-ph/9701231]. 